1 Hacettepe Journal of Mathematics and Statistics

$\bigcap$ Volume $44(2)$ (2015), $289-294$

\title{
Hyperconnectedness and extremal disconnectedness in (a)topological spaces
}

\author{
Arup Roy Choudhury ${ }^{*}$, Ajoy Mukharjee ${ }^{\dagger}$ and M. K. Bose ${ }^{\ddagger}$
}

\begin{abstract}
The aim of this paper is to study hyperconnectedness and extremal disconnectedness in a space equipped with a countable number of topologies.
\end{abstract}

2000 AMS Classification: Primary 54A10, Secondary 54D05, 54G05.

Keywords: (a)topological spaces, $(m, n)$ semiopen sets, hyperconnected (a)topological spaces, submaximal spaces, extremally disconnected (a)topological spaces.

Received 03 /12 /2011 : Accepted 31 /01 /2013 Doi : 10.15672/HJMS.2015449099

\section{Introduction}

A bitopological space [7] is a nonempty set with two topologies. Kovár [8, 9] also studied the properties of a nonempty set equipped with three topologies. Datta and Roy Choudhuri [5], and Raut and Datta [13] introduced nontrivial infinitesimally small elements. With this, they defined a number system as an extension of real number system. Their study offers a natural framework for dealing with an infinite sequence of distinct topologies on a set. Also emergence of chaos in a deterministic system, in the theory of dynamical system, relates to an interplay of finite or infinite number of different topologies in the underlining set. All these matters motivated the authors to consider a countable number of topologies in $(\omega)$ topological spaces $[2,3,4]$ and $\left(\aleph_{0}\right)$ topological spaces [1]. In this paper, we introduce the notion of (a)topological spaces which is a set equipped with countable number of topologies. The notion of $(a)$ topological spaces is more general than both the notions of $(\omega)$ topological spaces and $\left(\aleph_{0}\right)$ topological spaces.

*Department of Mathematics, Malda College, Malda, W. Bengal - 732 101, INDIA.

Email: roychoudhuryarup@yahoo.co.in

†Department of Mathematics, St. Joseph's College, Darjeeling, W. Bengal - 734 104, INDIA. Email: ajoyjee@yahoo.com, Corresponding Author.

${ }^{\ddagger}$ Department of Mathematics, University of North Bengal, Siliguri, W. Bengal-734 013, INDIA.

Email: manojkumarbose@yahoo.com 
Sarma [14] introduced the notion of pairwise extremal disconnectedness in bitopological spaces [7]. Among other results, she proved a result on pairwise extremal disconnectedness which is parallel to Urysohn's lemma on pairwise normal spaces. In [12], Mathew studied the hyperconnected topological spaces (Steen and Seebach [15]). In this paper, we study hyperconnectedness and extremal disconnectedness in the context of (a)topological spaces.

\section{2. (a)topological spaces}

2.1. Definition. If $\left\{\tau_{n}\right\}$ is a sequence of topologies on a set $X$, then the pair $\left(X,\left\{\tau_{n}\right\}\right)$ is called an (a)topological space.

If there is no scope of confusion, we denote the (a)topological space $\left(X,\left\{\tau_{n}\right\}\right)$, simply by $X$. Throughout the paper, $N$ denotes the set of natural numbers. The elements of $N$ are denoted by $i, j, k, l, m, n$ etc. If a set $G$ is open with respect to the topology $\tau_{n}$ i.e., if $G \in \tau_{n}$, then we say $G$ is $\left(\tau_{n}\right)$ open. $\left(\tau_{n}\right)$ closed set, $\left(\tau_{n}\right)$ closure have the obvious meaning. The $\left(\tau_{n}\right)$ closure (resp. $\left(\tau_{n}\right)$ interior) of a set $E$ is denoted by $\left(\tau_{n}\right) \mathrm{cl} E$ (resp. $\left.\left(\tau_{n}\right) \operatorname{int} E\right)$. Following Levine [11], we now introduce the following definitions.

2.2. Definition. Let $X$ be an (a)topological space and let $m \neq n$. A set $A \subset X$ is said to be $(m, n)$ semiopen if there exists a $U \in \tau_{m}$ such that $U \subset A \subset\left(\tau_{n}\right) \operatorname{cl} U$.

Thus any $\left(\tau_{m}\right)$ open set is an $(m, n)$ semiopen set for any $n \neq m$. The class of all $(m, n)$ semiopen sets with $m \neq n$, is denoted by $S O_{(m, n)}(X)$. We write $S O(X)=$ $\bigcup_{(m, n)} S O_{(m, n)}(X)$. A set belonging to $S O(X)$ is called an $(a)$ semiopen set.

2.3. Definition. An (a)topology $\left\{\sigma_{n}\right\}$ on $X$ is said to be stronger (resp. weaker) than an (a)topology $\left\{\tau_{n}\right\}$ if $\tau_{n} \subset \sigma_{n}$ (resp. $\sigma_{n} \subset \tau_{n}$ ) for each $n$. If, in addition, $\tau_{n} \neq \sigma_{n}$ for at least one $n$, then $\left\{\sigma_{n}\right\}$ is said to be strictly stronger (resp. weaker) than $\left\{\tau_{n}\right\}$.

2.4. Definition. An (a)topological space $\left(X,\left\{\tau_{n}\right\}\right)$ with property $P$ is said to be maximal (resp. minimal) with respect to $P$ if for any other (a)topology $\left\{\sigma_{n}\right\}$ strictly stronger (resp. weaker) than $\left\{\tau_{n}\right\}$, the space $\left(X,\left\{\sigma_{n}\right\}\right)$ can not have this property.

\section{Hyperconnected spaces}

Recall that a topological space $(X, \mathscr{T})$ is hyperconnected if the intersection of any two nonempty open sets is nonempty.

3.1. Definition. An (a)topological space $\left(X,\left\{\tau_{n}\right\}\right)$ is said to be hyperconnected if for any two nonempty sets $U$ and $V$ with $U \in \tau_{m}, V \in \tau_{n}$ and $m \neq n$, we have $U \cap V \neq \emptyset$.

It follows that if $X$ is hyperconnected, then for any nonempty set $U \in \tau_{m},\left(\tau_{n}\right) \operatorname{cl} U=X$ for any $n \neq m$.

3.2. Theorem. Suppose for any $\left(\tau_{m}\right)$ open set $G$ and $\left(\tau_{n}\right)$ open set $H, G \cap H \in \tau_{l}$ for some $l$. Then the (a)topological space $\left(X,\left\{\tau_{n}\right\}\right)$ and the topological spaces $\left(X, \tau_{n}\right), n \in N$ are hyperconnected iff the set $D$ of all nonempty $(a)$ semiopen sets is a filter.

Proof. Let the (a)topological space $\left(X,\left\{\tau_{n}\right\}\right)$ and the topological spaces $\left(X, \tau_{n}\right), n \in N$ be hyperconnected and $A, B \in D$. Then there exist two pairs $(m, n)$ and $(k, l)$ with $m \neq n$ and $k \neq l$ such that for some $U \in \tau_{m}$ and $V \in \tau_{k}, U \subset A \subset\left(\tau_{n}\right) \operatorname{cl} U, V \subset B \subset$ $\left(\tau_{l}\right) \mathrm{cl} V$. Since $\left(X,\left\{\tau_{n}\right\}\right)$ and $\left(X, \tau_{n}\right), n \in N$ are hyperconnected, we have $U \cap V \neq \emptyset$. Also $U \cap V \in \tau_{i}$ for some $i$. Therefore $\left(\tau_{j}\right) \operatorname{cl}(U \cap V)=X$ for any $j \neq i$. Hence $U \cap V \subset A \cap B \subset\left(\tau_{j}\right) \operatorname{cl}(U \cap V)$. Thus $A \cap B \in D$. Now let $A$ be a nonempty $(a)$ semiopen set and $B \supset A$. Then for some $(m, n)$ with $m \neq n$, there exists $U \in \tau_{m}$ such that 
$U \subset A \subset\left(\tau_{n}\right) \mathrm{cl} U$. But $\left(\tau_{n}\right) \operatorname{cl} U=X$. Therefore $U \subset B \subset\left(\tau_{n}\right) \operatorname{cl} U$. Hence $B$ is a nonempty $(a)$ semiopen set. Thus $D$ is a filter.

Since for any $n$, a $\left(\tau_{n}\right)$ open set is an $(a)$ semiopen set, the converse follows.

For a pair $(m, n)$, the union of an arbitrary number of $(m, n)$ semiopen sets is an $(m, n)$ semiopen set. If $X$ is hyperconnected, then the intersection of a finite number of $(m, n)$ semiopen sets is $(m, n)$ semiopen. Hence in this case, the class $S O_{(m, n)}(X)$ forms a topology on $X$. Since the class $\left\{S O_{(m, n)}(X) \mid m, n \in N\right\}$ is countable, and any countable class can be represented as a sequence, we rewrite the class $\left\{S O_{(m, n)}(X) \mid m, n \in N\right\}$ as a sequence $\left\{S_{k}\right\}$. So $\left(X,\left\{S_{k}\right\}\right)$ is an (a)topological space. From Theorem 3.2, it follows that if $\left(X,\left\{\tau_{n}\right\}\right)$ is hyperconnected and if for $G \in \tau_{m}$ and $H \in \tau_{n}, G \cap H \in \tau_{l}$ for some $l$, then $\left(X,\left\{S_{k}\right\}\right)$ is hyperconnected.

3.3. Corollary. If $\left(X,\left\{\tau_{n}\right\}\right)$ is maximal hyperconnected, then $\left\{\tau_{n}\right\}=\left\{S_{k}\right\}$.

Levine [10] introduced the concept of a simple extension of a topological space. Let $(X, \mathscr{P})$ be a topological space. A family $\mathscr{Q}$ of subsets of $X$ is a simple extension of $\mathscr{P}$ if $\mathscr{Q}$ contains $\mathscr{P}$ and there exists a $P \notin \mathscr{P}$ such that $\mathscr{Q}=\{G \cup(H \cap P) \mid G, H \in \mathscr{P}\}$.

3.4. Theorem. If for any $\left(\tau_{m}\right)$ open set $G$ and $\left(\tau_{n}\right)$ open set $H, G \cap H \in \tau_{l}$ for some $l$ and if the space $\left(X,\left\{\tau_{n}\right\}\right)$ is maximal hyperconnected and the space $\left(X, \tau_{n}\right), n \in N$ are hyperconnected, then the set $D$ of all nonempty (a) semiopen sets is an ultrafilter.

Proof. Since by Theorem 3.2, $D$ is a filter, it is sufficient to show that for a nonempty set $E, X-E \in D$ if $E \notin D$. Let us suppose that $E \notin D$. Then $E \notin \bigcup_{n} \tau_{n}$. Let $\tau_{n}(E)$ denote a simple extension of $\tau_{n}$. Then the space $\left(X,\left\{\tau_{n}(E)\right\}\right)$ which is stronger than $\left(X,\left\{\tau_{n}\right\}\right)$ is not hyperconnected. So for some nonempty set $U \in \tau_{m}(E)$ and nonempty set $V \in \tau_{n}(E)$ with $m \neq n$ we have $U \cap V=\emptyset$. By the definition of simple extension, $U=U_{1} \cup\left(U_{2} \cap E\right)$ and $V=V_{1} \cup\left(V_{2} \cap E\right)$ for some $U_{i} \in \tau_{m}$ and $V_{i} \in \tau_{n}, i=1,2$. Since $U \cap V=\emptyset, U_{1} \cap V_{1}=\emptyset$. But $\left(X,\left\{\tau_{n}\right\}\right)$ is hyperconnected and so either $U_{1}=\emptyset$ or $V_{1}=\emptyset$. Suppose without loss of generality, $U_{1}=\emptyset$. Now we consider the cases $(i) V_{1}=\emptyset$, and (ii) $V_{1} \neq \emptyset$.

Case $(i): V_{1}=\emptyset$. Since $U, V \neq \emptyset$, we have $U_{2} \neq \emptyset, V_{2} \neq \emptyset$. Therefore $U_{2} \cap V_{2} \neq \emptyset$, since $\left(X,\left\{\tau_{n}\right\}\right)$ is hyperconnected. Now

$$
\begin{array}{ll} 
& U \cap V=\emptyset \\
\Rightarrow & U_{2} \cap V_{2} \cap E=\emptyset \\
\Rightarrow & U_{2} \cap V_{2} \subset X-E \\
\Rightarrow \quad & X-E \in D, \text { since } D \text { is a filter. }
\end{array}
$$

Case $(i i): V_{1} \neq \emptyset$. Since $U_{2} \neq \emptyset$, we have $U_{2} \cap V_{1} \neq \emptyset$. Therefore $U_{2} \cap V_{1} \in D$. Again since $U \cap V=\emptyset$, we have $\left(U_{2} \cap E\right) \cap V_{1}=\emptyset \Rightarrow U_{2} \cap V_{1} \subset X-E$. Therefore $X-E \in D$. Thus $D$ is an ultrafilter.

Recall that a topological space $(X, \mathscr{T})$ is a door space if for each $A \subset X$, either $A \in \mathscr{T}$ or $X-A \in \mathscr{T}$.

3.5. Definition. The space $\left(X,\left\{\tau_{n}\right\}\right)$ is said to be a door space if for every subset $E$ of $X$, there exists an $n_{0}$ such that either $E \in \tau_{n_{0}}$ or $X-E \in \bigcup_{n \neq n_{0}} \tau_{n}$.

3.6. Theorem. If the (a)topological space $\left(X,\left\{\tau_{n}\right\}\right)$ is door and hyperconnected, then $\bigcap \tau_{n}-\{\emptyset\}$ is a filter.

Proof. Let $A, B \in \bigcap_{n} \tau_{n}-\{\emptyset\}$. Then $A, B$ are nonempty $\left(\tau_{n}\right)$ open sets for all $n$ and so $A \cap B \in \bigcap_{n} \tau_{n}$. Since $\left(X,\left\{\tau_{n}\right\}\right)$ is hyperconnected, $A \cap B \neq \emptyset$. Thus $A \cap B \in \bigcap_{n} \tau_{n}-\{\emptyset\}$. Now suppose $B \supset A \in \bigcap_{n} \tau_{n}-\{\emptyset\}$. Suppose that $B \notin \bigcap_{n} \tau_{n}-\{\emptyset\}$. Then $B \notin \tau_{n_{0}}-\{\emptyset\}$ 
for some $n_{0}$ and so $X-B \in \bigcup_{n \neq n_{0}} \tau_{n}$, since $\left(X,\left\{\tau_{n}\right\}\right)$ is door. So we have $A \in \tau_{n_{0}}$ and $A \cap(X-B)=\emptyset$, which contradicts the hyperconnectivity of $\left(X,\left\{\tau_{n}\right\}\right)$. Hence $B \in \bigcap_{n} \tau_{n}-\{\emptyset\}$. Therefore, $\bigcap_{n} \tau_{n}-\{\emptyset\}$ is a filter.

3.7. Theorem. If the (a)topological space $\left(X,\left\{\tau_{n}\right\}\right)$ is door and hyperconnected and the topological spaces $\left(X, \tau_{n}\right), n \in N$ are door $[6]$, then $\bigcap_{n} \tau_{n}-\{\emptyset\}$ is an ultrafilter.

Proof. By Theorem 3.6, $\bigcap_{n} \tau_{n}-\{\emptyset\}$ is a filter. If $E$ is a nonempty set with $E \notin \bigcap_{n} \tau_{n}-\{\emptyset\}$, then $E \notin \tau_{n_{0}}-\{\emptyset\}$ for some $n_{0}$. Then $X-E \in \tau_{n_{0}}$, since $\left(X, \tau_{n_{0}}\right)$ is door. Therefore, $E$ $\notin \bigcup_{n \neq n_{0}} \tau_{n}$, since $\left(X\left\{\tau_{n}\right\}\right)$ is hyperconnected. And so $E \notin \tau_{n}$ for all $n \neq n_{0} \Rightarrow X-E \in \tau_{n}$ for all $n \neq n_{0}$, since $\left(X, \tau_{n}\right), n \in N$ are door. Therefore $X-E \in \bigcap_{n} \tau_{n}-\{\emptyset\}$. Hence $\bigcap_{n} \tau_{n}-\{\emptyset\}$ is an ultrafilter.

3.8. Theorem. If $\left(X,\left\{\tau_{n}\right\}\right)$ is door and hyperconnected, then $\left(X,\left\{\tau_{n}\right\}\right)$ is minimal door and maximal hyperconnected.

Proof. Let $\left(X,\left\{\sigma_{n}\right\}\right)$ be a door space which is weaker than $\left(X,\left\{\tau_{n}\right\}\right)$. Suppose that $G \in \tau_{m}-\sigma_{m}$. Then $X-G \in \bigcup_{n \neq m} \sigma_{n} \subset \bigcup_{n \neq m} \tau_{n}$. But this is not possible, since $\left(X,\left\{\tau_{n}\right\}\right)$ is hyperconnected. Hence $\sigma_{n}=\tau_{n}$ for all $n$.

Now let $\left(X,\left\{\rho_{n}\right\}\right)$ be a hyperconnected space stronger than $\left(X,\left\{\tau_{n}\right\}\right)$. Suppose that $G \in \rho_{m}-\tau_{m}$. Then $X-G \in \bigcup_{n \neq m} \tau_{n} \subset \bigcup_{n \neq m} \rho_{n}$ which is not possible, since $\left(X,\left\{\rho_{n}\right\}\right)$ is hyperconnected. Thus $\rho_{n}=\tau_{n}$ for all $n$.

The following example shows that for an (a)topological door space $\left(X,\left\{\tau_{n}\right\}\right)$, the topological space $\left(X, \tau_{n}\right)$ may not be door even for a single $n$.

3.9. Example. Suppose $R$ is the set of real numbers. Let $\tau_{n}$ be the topology on $R$, generated by the subbase $\{\emptyset\} \cup\{E \subset(-\infty, n) \mid 0 \in E\} \cup\{E \subset R \mid 0 \in E$ and $E$ is not bounded above $\}$. Then the $(a)$ topological space $\left(X,\left\{\tau_{n}\right\}\right)$ is door but for no $n$, the topological space $\left(X, \tau_{n}\right)$ is door.

3.10. Definition. A set $E$ in an (a)topological space $\left(X,\left\{\tau_{n}\right\}\right)$ is said to be $(n \neq$ $\left.n_{0}\right)$ dense if $\bigcap_{n \neq n_{0}}\left(\tau_{n}\right) \operatorname{cl} E=X$.

3.11. Definition. The space $\left(X,\left\{\tau_{n}\right\}\right)$ is said to be submaximal if for any $n_{0}$, every $\left(n \neq n_{0}\right)$ dense subset is $\left(\tau_{n_{0}}\right)$ open.

3.12. Theorem. If the space $\left(X,\left\{\tau_{n}\right\}\right)$ is hyperconnected and submaximal, then it is maximal hyperconnected.

Proof. Let $\left(X,\left\{\sigma_{n}\right\}\right)$ be a hyperconnected space stronger than $\left(X,\left\{\tau_{n}\right\}\right)$. Let $G$ be a nonempty set belonging to $\sigma_{n_{0}}$ for some $n_{0}$. Then $\left(\sigma_{n}\right) \operatorname{cl} G=X$ for all $n \neq n_{0}$. Hence $\bigcap_{n \neq n_{0}}\left(\sigma_{n}\right) \operatorname{cl} G=X \Rightarrow \bigcap_{n \neq n_{0}}\left(\tau_{n}\right) \operatorname{cl} G=X$. Therefore $G$ is $\left(n \neq n_{0}\right)$ dense in $\left(X,\left\{\tau_{n}\right\}\right)$ and so $G \in \tau_{n_{0}}$. Hence $\tau_{n_{0}}=\sigma_{n_{0}}$. Thus $\tau_{n}=\sigma_{n}$ for all $n$.

\section{Extremally disconnected spaces}

4.1. Definition. An (a)topological space $\left(X,\left\{\tau_{n}\right\}\right)$ is said to be extremally disconnected if for any $G \in \tau_{m}$ and $H \in \tau_{n}$ with $G \cap H=\emptyset$ and $m \neq n$, there exist $k, l \in N$ with $m \neq k, n \neq l$ and $k \neq l$ such that for some $\left(\tau_{k}\right)$ closed set $F$ and $\left(\tau_{l}\right)$ closed set $K$, we have $G \subset F, H \subset K$ and $F \cap K=\emptyset$.

If the sequence $\left\{\tau_{n}\right\}$ consists of two topologies $\mathscr{P}$ and $\mathscr{Q}$ only, and if the bitopological space $(X, \mathscr{P}, \mathscr{Q})$ is pairwise extremally disconnected [14], then the space $\left(X,\left\{\tau_{n}\right\}\right)$ is extremally disconnected. 
4.2. Theorem. The (a)topological space $\left(X,\left\{\tau_{n}\right\}\right)$ is extremally disconnected iff for any $\left(\tau_{m}\right)$ open set $G$ and $\left(\tau_{n}\right)$ closed set $C$ with $G \subset C$ and $m \neq n$, there exist $k, l \in N$ with $m \neq k, n \neq l$ and $k \neq l$ such that for some $\left(\tau_{k}\right)$ closed set $F$ and $\left(\tau_{l}\right)$ open set $U$, we have $G \subset F \subset U \subset C$.

Proof. Suppose the space $X$ is extremally disconnected. Let us consider a $\left(\tau_{m}\right)$ open set $G$ and a $\left(\tau_{n}\right)$ closed set $C$ with $G \subset C$ and $m \neq n$. Then $X-C$ is a $\left(\tau_{n}\right)$ open set and $G \cap(X-C)=\emptyset$. Therefore there exist $k, l$ with $m \neq k, n \neq l$ and $k \neq l$ such that for some $\left(\tau_{k}\right)$ closed set $F$ and $\left(\tau_{l}\right)$ closed set $K$, we have $G \subset F, X-C \subset K$, $F \cap K=\emptyset \Rightarrow G \subset F \subset U \subset C$ where $U=X-K \in \tau_{l}$.

To prove the converse, suppose $G \in \tau_{m}$ and $H \in \tau_{n}$ with $G \cap H=\emptyset$ and $m \neq n$. Then $G \subset X-H$ and $X-H$ is $\left(\tau_{n}\right)$ closed. Therefore there exist $k, l$ with $m \neq k$, $n \neq l$ and $k \neq l$ such that for some $\left(\tau_{k}\right)$ closed set $F$ and $\left(\tau_{l}\right)$ open set $U$, we have $G \subset F \subset U \subset X-H$. If $K=X-U$, then $K$ is $\left(\tau_{l}\right)$ closed, $H \subset K$ and $F \cap K=\emptyset$. Thus the space is extremally disconnected.

4.3. Theorem. The space $\left(X,\left\{\tau_{n}\right\}\right)$ is extremally disconnected if for every $m$ and for every $G \in \tau_{m}$, we have $\left(\tau_{k}\right)$ cl $G \in \tau_{m}$ for all $k \neq m$.

Proof. Let $G \in \tau_{m}$ and $H \in \tau_{n}$ with $G \cap H=\emptyset$ and $m \neq n$. Then $\left(\left(\tau_{n}\right)\right.$ cl $\left.G\right) \cap H=\emptyset$. Since $m \neq n$, by the given condition we have $\left(\tau_{n}\right) \operatorname{cl} G \in \tau_{m}$. Therefore $\left(\left(\tau_{n}\right) \operatorname{cl} G\right) \cap\left(\left(\tau_{m}\right) \operatorname{cl} H\right)=$ $\emptyset$.

Let $\mathscr{T}$ denote the smallest topology on $X$ containing $\tau_{n}$ for all $n$.

4.4. Theorem. Let $\left(X,\left\{\tau_{n}\right\}\right)$ be extremally disconnected. Then for any $G \in \tau_{m}$ and $H \in \tau_{n}$ with $m \neq n$ and $G \cap H=\emptyset$, there exists a function $f: X \rightarrow[0,1]$ such that

(i) $f(G)=\{0\}, f(H)=\{1\}$

(ii) $f$ is $(\mathscr{T})$ continuous.

Proof. Since $G \subset X-H$ and $C=X-H$ is $\left(\tau_{n}\right)$ closed, by Theorem 4.2, there exist $k\left(\frac{1}{2}\right), l\left(\frac{1}{2}\right) \in N$ with $k\left(\frac{1}{2}\right) \neq m$ and $l\left(\frac{1}{2}\right) \neq n$ such that for some $\left(\tau_{k\left(\frac{1}{2}\right)}\right)$ closed set $F\left(\frac{1}{2}\right)$ and $\left(\tau_{l\left(\frac{1}{2}\right)}\right)$ open set $U\left(\frac{1}{2}\right)$ we have $G \subset F\left(\frac{1}{2}\right) \subset U\left(\frac{1}{2}\right) \subset C$. Again applying Theorem 4.2 to the pair $\left(G, F\left(\frac{1}{2}\right)\right)$ and $\left(U\left(\frac{1}{2}\right), C\right)$ there exist $k\left(\frac{1}{4}\right), l\left(\frac{1}{4}\right), k\left(\frac{3}{4}\right), l\left(\frac{3}{4}\right) \in N$ such that for some $\left(\tau_{k\left(\frac{1}{4}\right)}\right)$ closed set $F\left(\frac{1}{4}\right),\left(\tau_{k\left(\frac{3}{4}\right)}\right)$ closed set $F\left(\frac{3}{4}\right),\left(\tau_{l\left(\frac{1}{4}\right)}\right)$ open set $U\left(\frac{1}{4}\right)$ and $\left(\tau_{l\left(\frac{3}{4}\right)}\right)$ open set $U\left(\frac{3}{4}\right)$ we have $G \subset F\left(\frac{1}{4}\right) \subset U\left(\frac{1}{4}\right) \subset F\left(\frac{1}{2}\right) \subset U\left(\frac{1}{2}\right) \subset F\left(\frac{3}{4}\right) \subset U\left(\frac{3}{4}\right) \subset C$ and $k\left(\frac{1}{4}\right) \neq m, l\left(\frac{1}{4}\right) \neq k\left(\frac{1}{2}\right), k\left(\frac{3}{4}\right) \neq l\left(\frac{1}{2}\right), l\left(\frac{3}{4}\right) \neq n$. By repeating the process, we obtain $t \in D=\left\{\frac{i}{2^{j}} \mid 0<i<2^{j}, i, j \in N\right\}$, a $\left(\tau_{k(t)}\right)$ closed set $F(t)$ and a $\left(\tau_{l(t)}\right)$ open set $U(t)$ with $k(t), l(t) \in N$ such that if $s, t \in D$ with $s<t$, then $F(s) \subset U(s) \subset F(t) \subset U(t)$ and $k(t) \neq l(s)$. If we take $F(0)=\emptyset$ and $U(1)=X$, then the above relation is true when $s, t$ coincide with 0 or 1 . For $t \neq 0,1$, we have, $G \subset F(t) \subset U(t) \subset C$. Now we define the function $f: X \rightarrow[0,1]$ by $f(x)=\sup \{t \mid x \notin U(t)\}$. Then $f(x)=0$ for $x \in G$ and $f(x)=1$ for $x \in H$. It is easy to verify that for $a \in(0,1),\{x \in X \mid f(x)<$ $a\}=\bigcup_{t<a} U(t)$ and $\{x \in X \mid f(x)>a\}=\bigcup_{t>a}(X-F(t))$. Since $U(t) \in \tau_{l(t)} \subset \mathscr{T}$ and $X-F(t) \in \tau_{k(t)} \subset \mathscr{T}$, it follows that $f$ is $(\mathscr{T})$ continuous.

\section{Acknowledgement}

The authors are grateful to the referees for careful reading the paper and for certain suggestions towards the improvement of the paper. 


\section{References}

[1] Bose, M. K. and Mukharjee, A. On countable families of topologies on a set, Novi Sad J. Math. 40 (2), 7-16, 2010.

[2] Bose, M. K. and Tiwari, R. ( $\omega)$ topological connectedness and hyperconnectedness, Note Mat. 31, 93-101, 2011.

[3] Bose, M. K. and Tiwari, R. On ( $\omega$ )topological spaces, Riv. Mat. Univ. Parma. (7) 9, 125-132, 2008.

[4] Bose, M. K. and Tiwari, R. On increasing sequences of topologies on a set, Riv. Mat. Univ. Parma. (7) 7, 173-183, 2007.

[5] Datta, D. P. and Roy Choudhuri, A. Scale-free analysis and the prime number theorem, Fractals 18, 171-184, 2010.

[6] Kelley, J. L. General topology, Van Nostrand, Princeton, 1955.

[7] Kelly, J. C. Bitopological spaces, Proc. Lond. Math. Soc. 13, 71-89, 1963.

[8] Kovár, M. M. A note on the comparison of topologies, Int. J. Math. Math. Sci. 26, 233-237, 2001.

[9] Kovár, M. M. On 3-topological version of $\theta$-regularity, Int. J. Math. Math. Sci. 23, 393-398, 2000.

[10] Levine, N. Simple extensions of topologies, Amer. Math. Monthly 71, 22-25, 1964.

[11] Levine, N. Semi-open sets and semi-continuity in topological spaces, Amer. Math. Monthly 70, 36-41, 1963.

[12] Mathew P. M. On hyperconnected spaces, Indian J. Pure Appl. Math. 19, 1180-1184, 1988.

[13] Raut, S. and Datta, D. P. Non-archimedean scale invariance and cantor sets, Fractals 18, 111-118, 2010.

[14] Sarma, R. D. A note on extremal disconnectedness in bitopological spaces, Acta Math. Hungar. 120, 141-146, 2008.

[15] Steen, L. A. and Seebach (Jr), J. A. Counterexamples in Topology, Holt, Rinehart, and Winston, New York, 1970. 